\title{
Non-invasive algorithm for bowel motility estimation using a back-propagation neural network model of bowel sounds
}

\author{
Keo-Sik Kim ${ }^{1 \dagger}$, Jeong-Hwan Seo ${ }^{2 \dagger}$ and Chul-Gyu Song ${ }^{1,3^{*}}$
}

\author{
* Correspondence: \\ song133436@gmail.com \\ ${ }^{1}$ School of Electronics and \\ Information Engineering, Chonbuk \\ National University, 664-14 1 Ga, \\ Deokjin-dong, Jeonju, Republic of \\ Korea \\ Full list of author information is \\ available at the end of the article
}

\begin{abstract}
Background: Radiological scoring methods such as colon transit time (CTT) have been widely used for the assessment of bowel motility. However, these radiographbased methods need cumbersome radiological instruments and their frequent exposure to radiation. Therefore, a non-invasive estimation algorithm of bowel motility, based on a back-propagation neural network (BPNN) model of bowel sounds (BS) obtained by an auscultation, was devised.
\end{abstract}

Methods: Twelve healthy males (age: $24.8 \pm 2.7$ years) and 6 patients with spinal cord injury (6 males, age: $55.3 \pm 7.1$ years) were examined. BS signals generated during the digestive process were recorded from 3 colonic segments (ascending, descending and sigmoid colon), and then, the acoustical features (jitter and shimmer) of the individual BS segment were obtained. Only 6 features $\left(J_{1}, 3, J_{3,}, S_{1}, 2, S_{2}, 1, S_{2}, 2, S_{3}, 2\right)$, which are highly correlated to the CTTs measured by the conventional method, were used as the features of the input vector for the BPNN.

Results: As a results, both the jitters and shimmers of the normal subjects were relatively higher than those of the patients, whereas the CTTs of the normal subjects were relatively lower than those of the patients $(p<0.01)$. Also, through $k$-fold cross validation, the correlation coefficient and mean average error between the CTTs measured by a conventional radiograph and the values estimated by our algorithm were 0.89 and 10.6 hours, respectively.

Conclusions: The jitter and shimmer of the BS signals generated during the peristalsis could be clinically useful for the discriminative parameters of bowel motility. Also, the devised algorithm showed good potential for the continuous monitoring and estimation of bowel motility, instead of conventional radiography, and thus, it could be used as a complementary tool for the non-invasive measurement of bowel motility.

\section{Background}

Radiological scoring methods such as the Barr and Blethyn scores [1] and colon transit time (CTT) $[2,3]$, which operate by means of X-rays and magnetic resonance imaging (MRI), have generally been used for the assessment of bowel motility. Among these methods, the CTT described by Metcalf [2] is considered to be the gold-standard. It is simply assessed by measuring the movement of radiopaque markers taken at a fixed time after their ingestion. This test is highly reproducible and most useful in

(C) 2011 Kim et al; licensee BioMed Central Ltd. This is an Open Access article distributed under the terms of the Creative Commons Attribution License (http://creativecommons.org/licenses/by/2.0), which permits unrestricted use, distribution, and reproduction in any medium, provided the original work is properly cited. 
determining whether constipation symptoms are associated with normal or slow transit. However, these radiograph-based methods need an expensive, cumbersome radiological instrument and their frequent exposure to radiation.

In an effort to resolve these limitations, assessing bowel motility using bowel sound (BS) signals obtained by means of auscultation has been recently attempted. Tomomasa et al. [4] and Craine et al. [5,6] presented changes in various features (sound-/ motility-index, sound-to-sound interval, number of events and so on) of bowel sound according to the pathological condition. Yamaguchi et al. [7] showed that the sound index of the gastro-duodenal sound in the diabetes mellitus patients was significantly lower after food intake than in healthy adults. Also, wavelet-based strategies for the signal acquisition, de-noising, automated segmentation, event detection and feature extraction of bowel sounds were proposed [8-11]. Dimoulas et al. [12] implemented an autonomous BS monitoring system utilizing wavelet feature extraction and multi-layer perceptrons (MLP) network classifiers for the pattern classification of BS segments. Besides, the fractal-dimension analysis of BS signals $[9,10,13]$, principal component analysis (PCA) [14], Weiner filtering [15] and hybrid expert system using hierarchical audio pattern recognition [16] have been tried to detect the informative feature of BS and evaluate the bowel motility via an auscultation. These BSs are generated from the movement of the intestinal contents and gas in the lumen of the gastrointestinal tract during peristalsis; therefore, they can be used clinically as useful indicators of bowel motility.

Therefore, the aim of this study is to develop a non-invasive estimation algorithm of bowel motility, based on an artificial neural network (ANN) model of the jitter and shimmer, which were considered as useful features in recent study [17], of the BS signals during the digestive process. Also, we derived an ANN model between the acoustical features obtained from the BS signals and measured CTT, and finally determined the feasibility of the proposed method.

\section{Methods}

Twelve healthy men (age: $24.8 \pm 2.7$ years, BMI: $23.6 \pm 2.7 \mathrm{~kg} / \mathrm{m}^{2}$ ), not taking any medication that might affect their bowel motility, and 6 patients $(6$ men, age: $55.3 \pm$ 7.1 years, BMI: $24.0 \pm 3.5 \mathrm{~kg} / \mathrm{m}^{2}$ ) with delayed gastric emptying due to spinal cord injury were examined. The average number of months after injury was $17.2 \pm 28.5$ months. Ethical approval for this study was obtained from the Institutional Review Board (IRB) of Chonbuk National University Hospital.

The total CTTs were measured by the strategy described by Metcalf et al. [2]. The subject ingested a radiopaque marker capsule containing 20 markers (Kolomark ${ }^{\mathrm{TM}}$, Korea) each day for 3 days. On days 4 and 7, an abdominal X-ray image was obtained. Also, on day 7, the 3-channel BS signals were obtained from the right upper (ascending colon, $\mathrm{CH} 1$ ), left upper (descending colon, $\mathrm{CH} 2$ ) and left lower quadrants (sigmoid colon, $\mathrm{CH} 3$ ) of the abdomen with the subjects lying on a bed, respectively. Under fasting conditions, the subjects took test meals of $200 \mathrm{~g}$ at 9:00 AM. After that, the data was recorded for 10 minutes at 9:30 AM (Post1), 1:00 PM (Post4) and 5:00 PM (Post8). The subjects were asked to take no food from 9:30 AM to 5:00 PM.

A piezo-polymer microphone (CM-01B, Measurement Specialties Inc., U.S.) with a frequency bandwidth of 8-2, $200 \mathrm{~Hz}$ was used for collecting the BS signal. The frequency 
content of the BS signals is known to be energetic mainly between 100 and $500 \mathrm{~Hz}$ $[11,18]$, thus the collected BS signals were pre-processed through a $60 \mathrm{~Hz}$ notch filter for removing the power noise and 5-600 Hz band-pass filter for reducing the motion artefact noise caused by respiration activation and unwanted noise. After that, the signals were digitized by an A/D converter (USB-6009, National Instruments ${ }^{\text {TM }}$, U.S.) at a sampling rate of $8 \mathrm{KHz}$ and resolution of 14 bits. Figure 1 shows the sensor-adaptation set-up of the noncontact-type probe used for recording BS signals.

The statistical analysis was conducted using SPSS (ver. 14, SPSS Inc., U.S.) software. Unpaired T-test was performed to compare the features obtained from the normal subjects with those from the patients. Also, Pearson's correlation coefficients were obtained to evaluate the relationship between the acoustical features by means of our algorithm and CTT by means of the conventional method. The level of statistical significance was set as $p<0.05$. Finally, $k$-fold cross-validation was performed to evaluate the performance of our algorithm.

\section{Bowel motility estimation}

\section{BS detection and segmentation}

Figure 2 shows the procedure used for detecting selectively the BS segments from noisy BS signals and extracting the features, in order to estimate the CTT using the ANN model. First, the recorded BS signals were processed using the modified iterative kurtosis-based detection (mIKD) algorithm, devised in our previous study [17], for the selective detection of BS segments through noise-gating. Significant deviations from kurtosis value can be attributed to the presence of non-Gaussian signals such as the BS, since kurtosis is theoretically zero for Gaussian signals such as back-ground sound

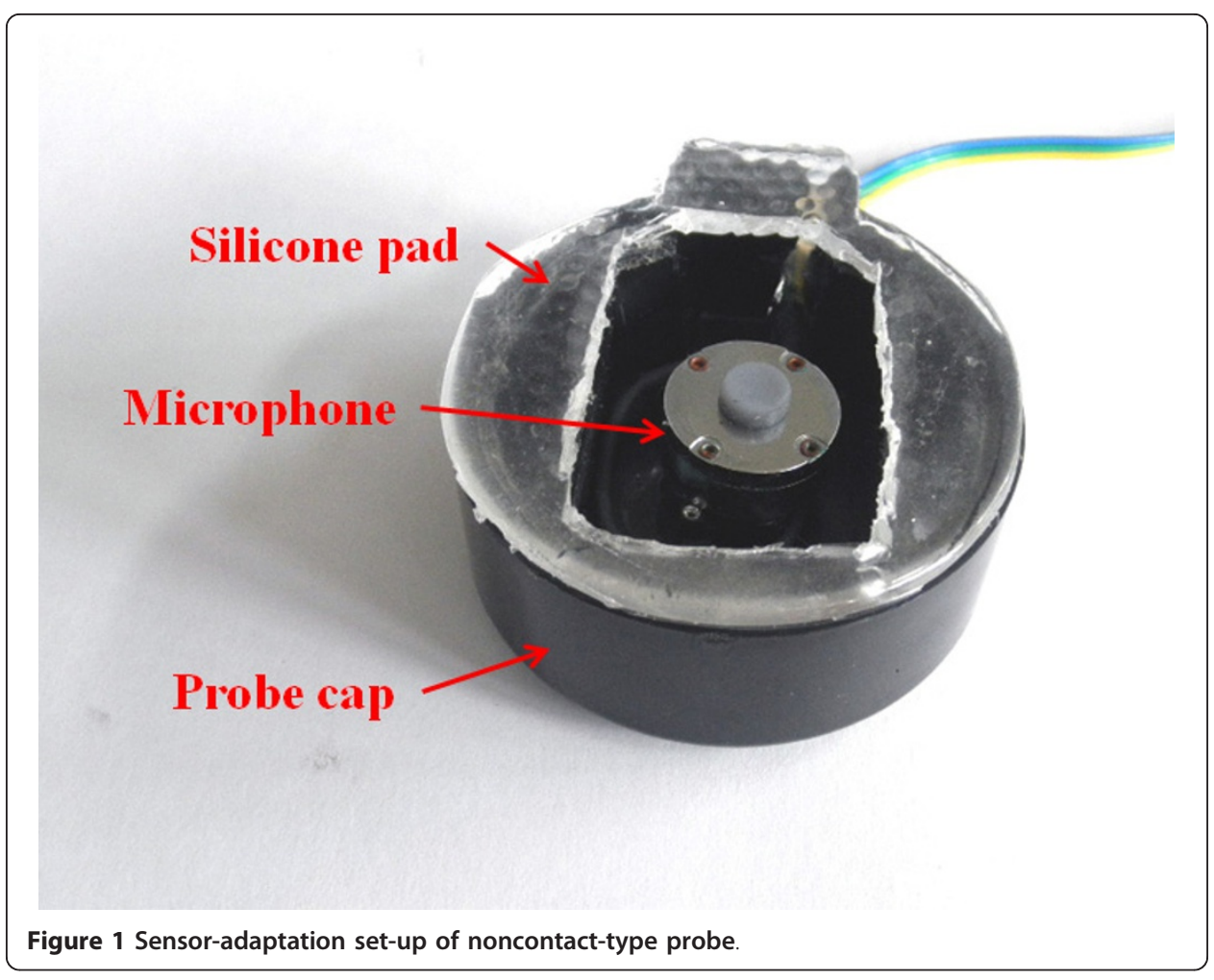




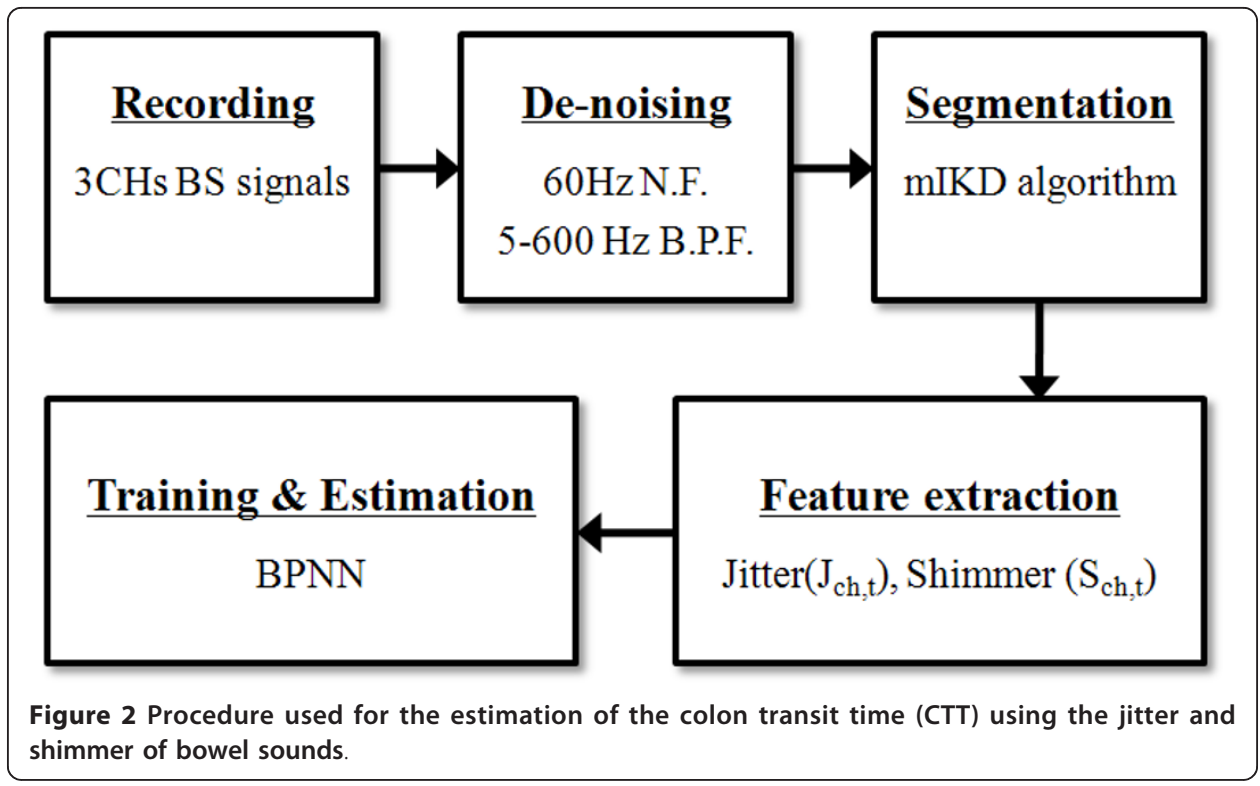

(BGSs) signals [19]. Next, the detected BS signals were divided into the individual BS segments, where an individual BS segment is defined as sounds having duration larger than $20 \mathrm{msec}$ without the period of silence [11].

\section{Feature extraction}

The characteristic parameters, viz. the absolute jitter $\left(J_{c h, t}\right)$ and shimmer $\left(S_{c h, t}\right)$, of each BS segment, were calculated using (1) and (2), respectively;

$$
\begin{aligned}
& J_{c h, t}=\frac{1}{N-1} \sum_{i=1}^{N-1}\left|\frac{1}{P_{i}}-\frac{1}{P_{i+1}}\right| \\
& S_{c h, t}=\frac{1}{N-1} \sum_{i=1}^{N-1}\left|20 \log \left(A_{i+1} / A_{i}\right)\right|
\end{aligned}
$$

where $P_{i}, A_{i}$ and $N$ are the peak-to-peak period and amplitude of each pitch and the number of pitches, while $c h$ and $t$ are the channel number $(1=\mathrm{CH} 1,2=\mathrm{CH} 2$ and 3 $=\mathrm{CH} 3)$ and time index $(1=$ Post $1,2=$ Post 4 and $3=$ Post 8$)$ when the BSs were recorded, respectively. The jitter is the average absolute difference between consecutive periods, while the shimmer is the average absolute difference between the amplitudes of consecutive periods in decibels [20].

A total of 18 features (the $J_{c h, t}$ of 9 and $S_{c h, t}$ of 9) per subject were obtained. Among all of these 18 features, the features highly correlated with the measured CTT were selected as the informative one through correlation analysis. As a result, only the top six features $\left(J_{1,3}, S_{1,2}, S_{3,2}, J_{3,3}, S_{2,2}, S_{2,1}\right)$ with the high correlation coefficient of 0.65 and over were used as the features of the input vector for the ANN. Table 1 represents the correlation coefficients between the selected features and CTT and their $p$-values.

\section{Architecture of the ANN}

The estimation of the CTT was performed using a back-propagation neural network (BPNN). The input and output layers of the BPNN consisted of 7 nodes (selected 6 
Table 1 Correlation coefficients (C.C.) between the selected features and measured colon transit time (CTT) and their $p$-values

\begin{tabular}{|c|c|c|c|c|c|c|c|}
\hline & $J_{1,3}$ & $S_{1,2}$ & $S_{3,2}$ & $J_{3.3}$ & $S_{2,2}$ & $S_{2,1}$ & CTT \\
\hline N1 & 0.154 & 37.6 & 36.1 & 0.159 & 35.1 & 35.4 & 10.8 \\
\hline N2 & 0.172 & 34.4 & 34.2 & 0.167 & 35.4 & 35.6 & 26.4 \\
\hline N3 & 0.176 & 38.3 & 36.6 & 0.177 & 38.2 & 37.0 & 10.8 \\
\hline N4 & 0.173 & 34.7 & 32.4 & 0.166 & 35.1 & 33.8 & 2.4 \\
\hline N5 & 0.162 & 34.6 & 36.8 & 0.155 & 36.3 & 38.3 & 18.0 \\
\hline N6 & 0.164 & 34.6 & 34.1 & 0.168 & 35.2 & 35.0 & 12.0 \\
\hline N7 & 0.160 & 37.1 & 36.5 & 0.164 & 34.7 & 35.2 & 16.8 \\
\hline N8 & 0.161 & 34.5 & 35.0 & 0.164 & 35.7 & 37.5 & 60.0 \\
\hline N9 & 0.157 & 36.2 & 33.8 & 0.168 & 35.4 & 37.3 & 26.4 \\
\hline N10 & 0.159 & 36.0 & 36.0 & 0.161 & 34.6 & 38.2 & 25.2 \\
\hline N11 & 0.167 & 35.0 & 36.7 & 0.165 & 34.3 & 36.4 & 2.4 \\
\hline N12 & 0.163 & 32.3 & 32.3 & 0.161 & 35.8 & 33.1 & 33.0 \\
\hline P1 & 0.132 & 30.2 & 31.1 & 0.151 & 30.1 & 29.8 & 69.0 \\
\hline P2 & 0.139 & 32.0 & 27.8 & 0.148 & 32.6 & 31.9 & 86.0 \\
\hline P3 & 0.147 & 31.8 & 30.6 & 0.154 & 31.7 & 32.4 & 102.0 \\
\hline P4 & 0.149 & 30.9 & 34.5 & 0.156 & 35.0 & 31.9 & 66.0 \\
\hline P5 & 0.134 & 32.5 & 30.8 & 0.142 & 30.7 & 30.5 & 82.2 \\
\hline P6 & 0.155 & 29.9 & 30.9 & 0.155 & 30.5 & 30.7 & 68.0 \\
\hline C.C. & $-0.79^{*}$ & $-0.76^{*}$ & $-0.75^{*}$ & $-0.74^{*}$ & $-0.72^{*}$ & $-0.68^{*}$ & \\
\hline
\end{tabular}

$\mathrm{N}$ : normal subject, $\mathrm{P}$ : patient, $\mathrm{J}_{\mathrm{ch}, \mathrm{t}}:$ jitter, $\mathrm{S}_{\mathrm{ch}, \mathrm{t}}$ : shimmer, ${ }^{*}: p<0.01$

features and 1 bias) and 1 node (estimated CTT, eCTT), respectively. The training of a network by back-propagation involves three stages; the feed forward of the input training pattern, the calculation and back-propagation of the associated error, and the adjustment of the weights. After training, the application of the net involves only the computations of the feed-forward phase [21].

Also, the performance of a BPNN can depend on its structure such as the learning rate and number of hidden nodes. Thus, we determined their practical values which can provide the best performance, i.e. the least error and best correlation between the measured CTT and estimated one. The values of learning rate tested were 0.05, 0.1, $0.2,0.3$ and 0.4 ( 5 cases), and the values of the number of hidden nodes were $2,3,4$ and 5 ( 4 cases).

\section{Results}

Figure 3 shows (a) the raw signals obtained from healthy subject (31-year-old male, ascending colon at Post1), (b) BS segments detected by the mIKD algorithm and (c) background noise. As shown in the output of the mIKD (in Figure 3b), our algorithm could sensitively separate even the low peaks (e.g. around time 19.5 seconds) although they were hard to be distinguished from BGSs by means of visual or auditory inspection. On the contrary, the high peak (e.g. around 21.5 seconds) was classified as BGS since the kurtosis value of the corresponding segment was almost zero. These results show the performance of the mIKD algorithm used for selectively detecting inherent BS segments, despite the difference in the BGS level and in BSs amplitude and number.

Firstly, in order to determine the availability of the jitter and shimmer used in our algorithm, we compared the values of the selected features obtained from the normal 

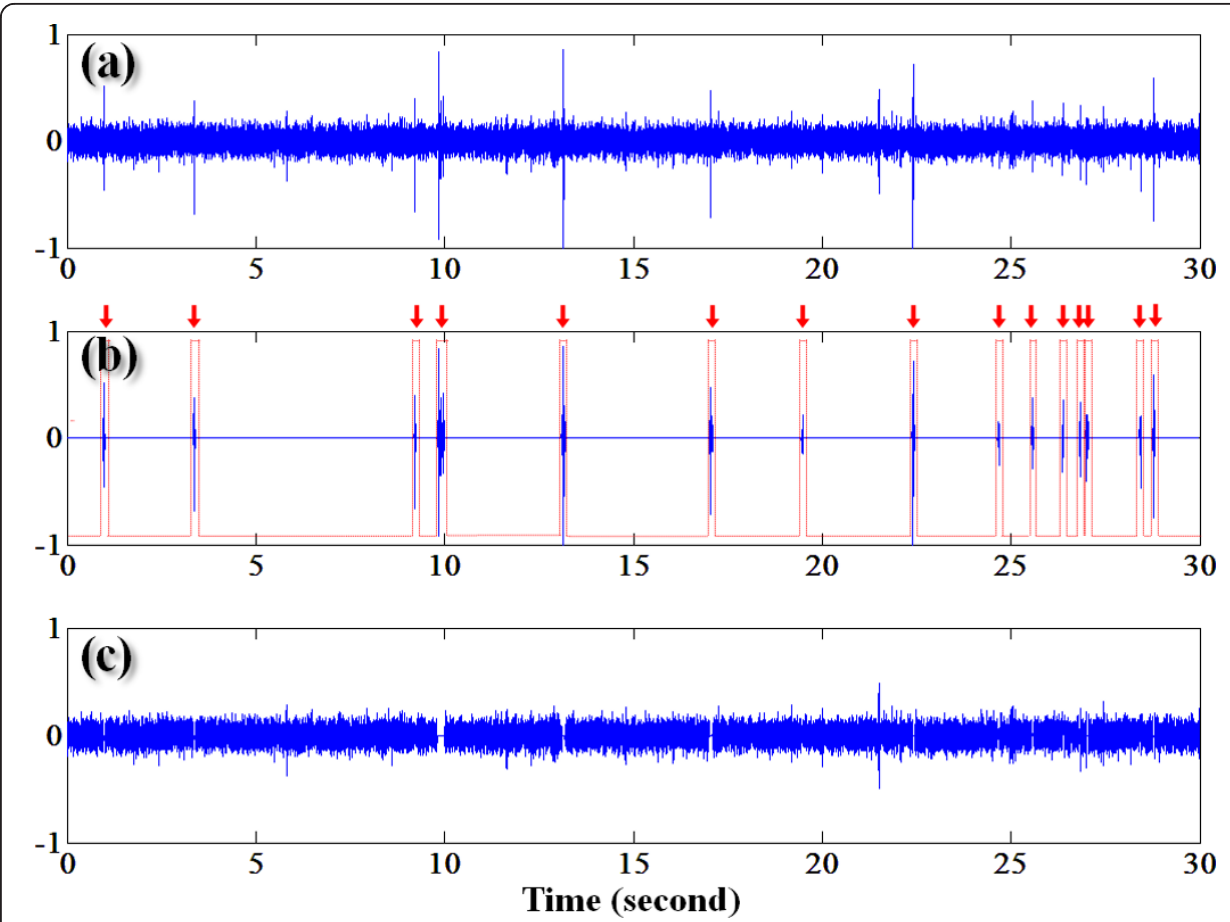

Figure 3 a) Raw bowel sound signals obtained from the abdomen. b) Inherent bowel sounds detected by the modified iterative kurtosis-based detector algorithm. c) Background noise. Arrows represent the individual bowel sound segments.

subjects with those of the patients. As a result, both the jitters $\left(J_{1,3}: 0.164 \pm 0.007, J_{3}\right.$, $3: 0.165 \pm 0.006)$ and shimmers $\left(S_{1,2}: 35.4 \pm 1.7, S_{3,2}: 35.0 \pm 1.7, S_{2,2}: 35.5 \pm 1.0, S_{2}\right.$, 1: $36.1 \pm 1.7 \mathrm{~dB}$ ) of the normal subjects were relatively higher than those of the patients $\left(J_{1,3}: 0.143 \pm 0.009, J_{3,3}: 0.151 \pm 0.005, S_{1,2}: 31.2 \pm 1.0, S_{3,2}: 30.9 \pm 2.1, S_{2,2}\right.$ : $\left.31.8 \pm 1.8, S_{2,1}: 31.2 \pm 1.0 \mathrm{~dB}\right)$, whereas the CTTs of the normal subjects $(20.4 \pm 15.8$ hours) were relatively lower than those of the patients (78.9 \pm 14.0 hours) $(p<0.01)$.

Next, for evaluating the performance of our algorithm, $k$-fold cross-validation $(k=3)$ was done using WEKA machine learning software (ver. 3.6. The University of Waikato, New Zealand) [22]. After the random rearrangement of all of 18 feature-datasets, $67 \%$ of them (12 feature-datasets) were used for training the BPNN model and the remaining 33\% (6 feature-datasets) were used for estimating the CTT using the model trained previously. Consequently, the correlation coefficient, mean average error (MAE) and root mean square error (RMSE) between the measure CTTs and estimated values (eCTT) were $0.89,10.6$ and 14.6 hours at the number of epoch of 5,900, respectively, when applying the learning rate of 0.05 and the number of hidden nodes of 3 to the designed BPNN. Figure 4 shows the changes of the correlation coefficient and MAE of the testing-datasets according to the increase of the number of epoch.

\section{Discussion}

Auscultation of the abdomen, one of physical examinations, has been used as a traditional technique for evaluating bowel motility. For the last few decades, there have been various comparative studies on the BSs of normal subjects and those of patients with bowel dysfunction, such as irritable bowel syndrome [5,6,8], Crohn's disease [6], 


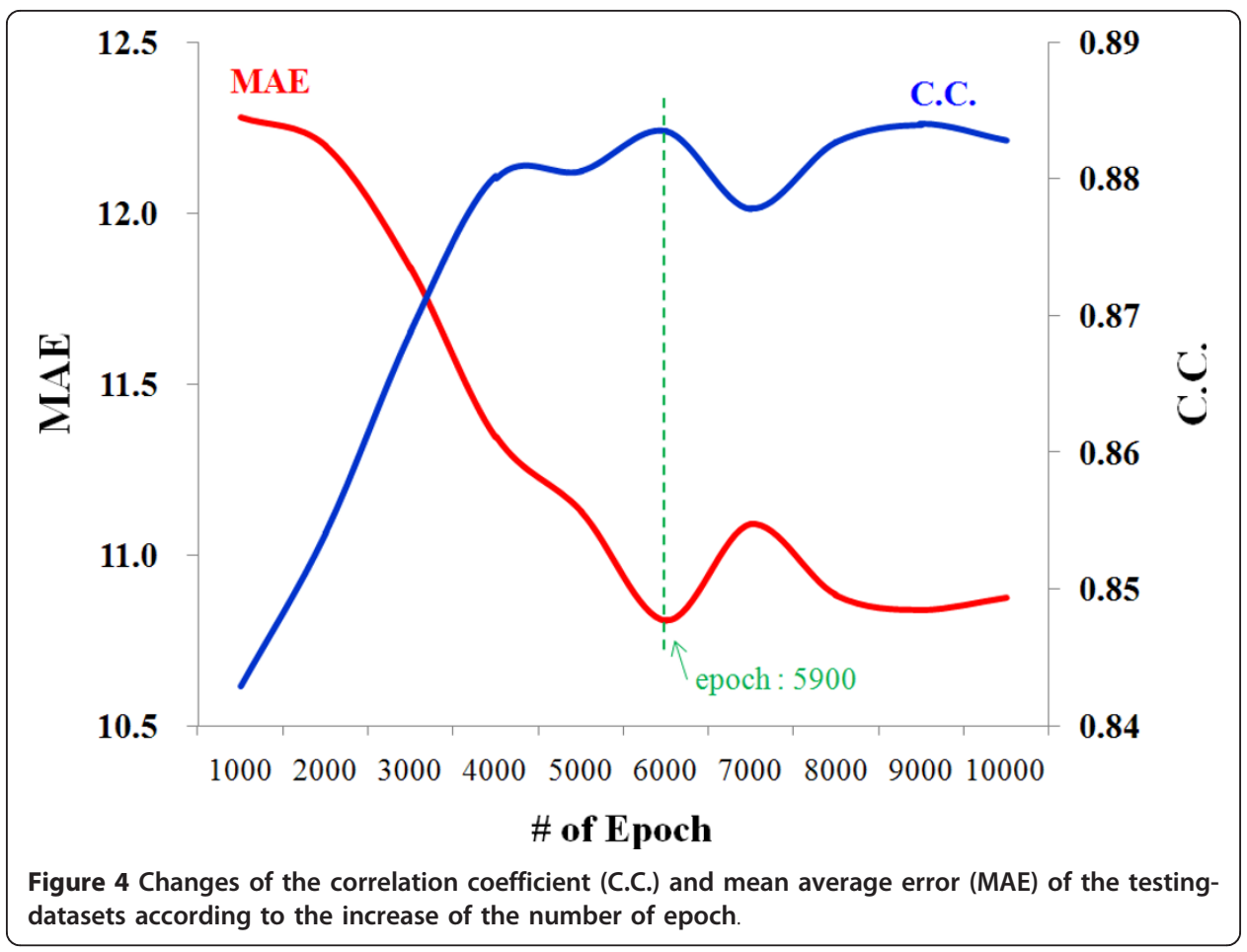

diabetes mellitus [7] and obstruction [18], and their results showed significant differences between the features obtained from BS signals according to the pathological condition of bowel motility. Also, several researchers recently have developed the denoising, segmentation and feature extraction strategies of BS signals based on various signal-processing techniques $[9-16,19]$. However, relatively few studies related to the quantitative estimation of bowel motility have been performed. Therefore, the goal of this study is to show the possibility of the quantitative estimation of the CTT, which has been used for the clinical assessment of bowel motility, by means of an ANN model and acoustical features.

BSs are produced from the movement of the intestinal contents, gas and fluid during peristalsis. Generally, normal 'very loud', 'gurgling' and 'rumbling' sounds are easily produced by a healthy bowel during an active stage of digestion. On the other hands, hyperactive BSs ('loud', 'high-pitched' and 'tinkling' sounds) might be caused by diarrhea or early intestinal obstruction, whereas hypoactive sounds (very diminished or absent sounds) are associated with bowel obstruction, paralytic ileus, torsion of the bowel or peritonitis that indicate diminished peristalsis [23,24]. As considering these differences of sounds, several informative BS-features related to the pathological condition of the gastrointestinal tract have been reported: time-domain features, such as sound-to-sound interval (silence between BSs durations) [5,6,14], number of BSs by time interval $[4,6,11,14]$, sound index (sum of the BSs amplitude) [4,7], median duration $[11,14,16]$ and median acoustic intensity $[11,14]$ of BSs, and frequency-domain ones, such as main frequency of BSs $[4,11,14,16,18]$.

Besides those features mentioned above, jitter and shimmer selected in our approach are measures of the fundamental frequency and amplitude cycle-to-cycle variations, respectively. They can represent the deviation or displacement of some aspect of the 
pitches of sounds in frequency- and time-domain, accordingly, they have been successfully used in a speaker verification, emotion expression, vocal or articular pathologies [20,25-27]. In this study, the jitters and shimmers were significantly different between two groups $(p<0.01)$ and highly correlated with the CTTs (correlation coefficient from -0.68 to -0.79 ). These differences were thought to be related to the delayed peristalsis caused by the impaired vagus and hypogastric nerves of the SCI patients [28], and this aspect might produce relatively decreased perturbation of the pitches of BSs. Therefore, the jitter and shimmer considered in our method could be employed as a useful clinical parameter for the continuous monitoring of the bowel motility.

In relation to recording-duration issues of our approach, the BS signals were analyzed based on short-term analysis that deals with small-duration samples of the entire activity in fasted humans, coincided with $[5-8,18,19]$, and all of the features were obtained from impulsive BSs due to the management difficulty of subject's immobilization during the test and huge data. On the contrary, many researchers believe that the period of BS monitoring should be longer over at least 2 hours since lasting/regularlysustained (RS) BSs monitored during a full migrating motor complex (MMC) cycle are also associated with bowel motility [4,10-16]. Unfortunately, even though the acoustical features obtained from the short-term recording of 10 minutes set in this study showed its feasibility, this strategy would raise a subject prolific of controversy related to the statistical reliability of the acquired samples. Therefore, in a future study we need to determine the appropriate recording period and to implement additional techniques for RS-BSs treatment as well as impulsive sounds.

Recently, the estimation method based on the regression analysis between the features obtained from BS signals, such as the jitter and shimmer, and conventional CTT was implemented in [17]. Accordingly, the comparison of the estimation results of the proposed approach by means of the ANN model with those obtained from the previous regression model was performed. When applying 18 feature-datasets used in this study into the regression model, the regression equation between the selected features and measured CTT was obtained as follows;

$$
e C T T=430.3-1462.9 J_{1,3}-5.7 S_{1,2}-4.3 S_{3,2}+261.1 J_{3,3}+0.8 S_{2,2}+3.1 S_{2,1}
$$

Also, the correlation coefficient, MAE and RMSE between the CTTs and eCTTs were $0.89,12.4$ and 18.4 hours, respectively. As a result, the estimation errors of the ANN model (MAE of 10.6 and RMSE of 14.6 hours) were relatively lower than those of the regression model, whereas the correlation coefficients of both methods were almost same. Consequently, these results showed that the neural-network-based approach attempted in this study could enhance its performance.

The limitations of this study are the small number of subjects, and the effects of the physiological characteristics of the subjects, such as their body mass index or severity of the spinal cord impairment were not considered in our algorithm. In a future study, we will apply our algorithm to a larger number of patients with various bowel dysfunctions, as well as to normal subjects, in order to enhance the accuracy of the estimated CTT. Also, we plan to develop supplementary signal processing techniques for effectively reducing frictional noise generated unavoidably between the skin and microphone as well as unwanted bio-signals. 


\section{Conclusions}

A non-invasive algorithm for the estimation of the CTT based on BPNN model of the jitter and shimmer of the BS signals obtained by auscultation is reported. The correlation coefficient and MAE between the CTTs measured by the conventional method and the values estimated by our algorithm were 0.89 and 10.6 hours, respectively. The proposed algorithm showed good potential for the non-invasive measurement and continuous monitoring of bowel motility, instead of conventional radiography.

\section{List of Abbreviations}

CTT: Colon Transit Time; MRI: Magnetic Resonance Imaging; BS: Bowel Sound; MLP: Multi-Layer Perceptron; ANN: Artificial Neural Network; IRB: Institutional Review Board; mIKD: Modified Iterative Kurtosis-based Detection; BGS: BackGround Sound; NF: Notch Filter; BPF: Band Pass Filter; BPNN: Back-Propagation Neural Network; eCTT: Estimated Colon Transit Time; MAE: mean average error; RMSE: root mean square error; MMC: Migrating Motor Complex.

\section{Acknowledgements \\ This work was supported by the Human Resources Development of the Korea Institute of Energy Technology Evaluation and Planning (KETEP) grant funded by the Korea Government Ministry of Knowledge Economy (No.20104010100660) and supported by the National Research Foundation of Korea Grant funded by the Korean Government(MEST) (NRF-2010-0021864).}

\section{Author details}

School of Electronics and Information Engineering, Chonbuk National University, 664-14 1 Ga, Deokjin-dong, Jeonju, Republic of Korea. 'School of Medicine, Department of Rehabilitation Medicine, Chonbuk National University Hospital, 634-18 Geumam-dong, Jeonju, Republic of Korea. ${ }^{3}$ Center for Advanced Image and Information Technology, Chonbuk National University, 664-14 1 Ga, Deokjin-dong, Jeonju, Republic of Korea.

\section{Authors' contributions}

KK devised an estimation algorithm for bowel motility and implemented the portable system which consists of preprocessing circuits and data acquisition/analysis software using LabVIEW language. JS contributed to the experimental protocol for clinical assessment of bowel motility and interpretation of the results. CS supervised the project and contributed to the design of the algorithms. All authors read and approved the final manuscript.

\section{Competing interests}

The authors declare that they have no competing interests.

Received: 12 April 2011 Accepted: 10 August 2011 Published: 10 August 2011

\section{References}

1. Jackson CR, Lee RE, Wylie ABZ, Adams C, Jaffray B: Diagnostic accuracy of the Barr and Blethyn radiological scoring systems for childhood constipation assessed using colonic transit time as the gold standard. Pediatr Radiol 2009, 39:664-667.

2. Metcalf AM, Phillips SF, Zinmeister AR, MacCarty RJ, Beart RW, Wolff BG: Simplified assessment of segmental colonic transit. Gastroenterology 1987, 92:40-47.

3. Emmanuel A, Roy A: Small intestine and colon motility. Medicine 2007, 35:272-276.

4. Tomomasa T, Morikawa A, Sandler R, Mansy H, Koneko H, Masahiko T, Hyman P, Itoh Z: Gastrointestinal sounds and migrating motor complex in fasted humans. Am J Gastroenterol 1999, 94:374-381.

5. Craine BL, Silpa ML, OToole CJ: Computerized auscultation applied to irritable bowel syndrome. Dig Dis Sci 1999, 44:1887-1892.

6. Craine BL, Silpa ML, OToole CJ: Enterotachogram analysis to distinguish irritable bowel syndrome from Crohn's disease. Dig Dis Sci 2001, 46:1974-1979.

7. Yamaguchi K, Yamaguchi T, Odaka T, Saisho H: Evaluation of gastrointestinal motility by computerized analysis of abdominal auscultation findings. J Gastroenterol Hepatol 2006, 21:510-514.

8. Hadjileontiadis LJ, Liatsos CN, Mavrogiannis CC, Rokkas TA, Panas SM: Enhancement of bowel sounds by waveletbased filtering. IEEE Trans Biomed Eng 2000, 47:876-886.

9. Hadjileontiadis LJ: Wavelet-Based Enhancement of Lung and Bowel Sounds Using Fractal Dimension ThresholdingPart I: Methodology. IEEE Trans Biomed Eng 2005, 52:1143-1148.

10. Dimoulas C, Kalliris G, Papanikolaou G, Kalampakas A: Long term signal detection, segmentation and summarization using wavelets and fractal dimension: a bioacoustics application in gastrointestinal motility monitoring. Comput Biol Med 2007, 37:438-462.

11. Ranta R, Dorr VL, Heinrich C, Wolf D, Guillemin F: Digestive activity evaluation by multichannel abdominal sounds analysis. IEEE Trans Biomed Eng 2010, 57:1507-1519.

12. Dimoulas C, Kalliris G, Papanikolaou G, Petridis V, Kalampakas A: Bowel-sound pattern analysis using wavelets and neural networks with application to long-term, unsupervised, gastrointestinal motility monitoring. Expert Syst Appl 2008, 36:26-41.

13. Hadjileontiadis $L J$, Rekanos IT: Detection of explosive lung and bowel sounds by means of fractal dimension. IEEE Signal Process Lett 2003, 10:311-314. 
14. Ranta R, Louis-Dorr V, Heinrich C, Wolf D, Guillemin F: Principal component analysis and interpretation of bowel sounds. Proceeding of the 26th Annual International Conference of the IEEE EMBS 2004, 227-230.

15. Dimoulas CA, Kalliris G, Papanikolaou G, Kalampakas A: Novel wavelet domain Weiner filtering de-noising techniques: Application to bowel sounds captured by means of abdominal surface vibrations. Biomed Signal Process Control 2006, 1:177-218.

16. Dimoulas CA, Papanikolaou G, Petridis V: Pattern classification and audiovisual content management techniques using hybrid expert systems: A video-assisted bioacoustics application in abdominal sounds pattern analysis. Expert Syst Appl 2011, 38:13082-13093.

17. Kim KS, Seo JH, Ryu SH, Kim MH, Song CG: Estimation algorithm of the bowel motility based on regression analysis of the jitter and shimmer of bowel sounds. Comput Methods Programs Biomed

18. Yoshino H, Abe Y, Yoshino T, Oshato K: Clinical application of spectral analysis of bowel sounds in intestinal obstruction. Dis Colon Rectum 1990, 33:753-757.

19. Rekanos IT, Hadjileontiadis $L$ : An iterative kurtosis-based technique for the detection of nonstationary bioacoustic signals. Signal Process 2006, 86:3787-3795.

20. Farrus M, Hernando J: Using jitter and shimmer in speaker verification. IET signal Process 2009, 3:247-257.

21. Fausett L: Fundamentals of Neural Networks: Architectures, Algorithms and Applications Prentice-Hall Inc; 1994.

22. Weka 3: Data mining software in java. [http://www.cs.waikato.ac.nz/ ml/weka/].

23. Javis C: Physical Examination and Health Assessment Elsevier; 2008.

24. Baid H: A critical review of auscultating bowel sounds. Br J Nurs 2009, 18:1125-1129.

25. Baken R, Orlikoff RF: Clinical Measurements of Speech and Voice Thomson Delmar Learning; 2000.

26. Ayadi ME, Kamel MS, Karray F: Survey on speech emotion recognition: Features, classification schemes, and databases. Pattern Recognit 2011, 44:572-587.

27. Kim KS, Seo JH, Song CG: An acoustical evaluation of knee sound for non-invasive screening and early detection of articular pathology. J Med Syst.

28. Lynch AC, Antony A, Dobbs BR, Frizelle FA: Bowel dysfunction following spinal cord injury. Spinal Cord 2001, 39:193-203.

doi:10.1186/1475-925X-10-69

Cite this article as: Kim et al: Non-invasive algorithm for bowel motility estimation using a back-propagation neural network model of bowel sounds. BioMedical Engineering OnLine 2011 10:69.

\section{Submit your next manuscript to BioMed Central and take full advantage of:}

- Convenient online submission

- Thorough peer review

- No space constraints or color figure charges

- Immediate publication on acceptance

- Inclusion in PubMed, CAS, Scopus and Google Scholar

- Research which is freely available for redistribution 